\title{
Merck's new drug free to WHO for river blindness programme
}

Washington

IF a newly-launched programme to eradicate onchocerciasis, or river blindness, a parasitic infestation affecting as many as 20 million people in Africa, south and central America and the Middle East, is successful, much of the credit will be due to Merck \& Co., the New Jersey pharmaceutical company, which announced last week that it will provide its antiparasitic drug Mectizan free of charge to countries that need it. The burden of distributing the drug and supervising medical care falls to the World Health Organisation (WHO), but Merck was apparently the initiator of the idea and its driving force until WHO was persuaded of the potential effectiveness of the new treatment.

The parasitic worm Onchocerca volvulus is transmitted to man by the blackfly, which breeds in fast-flowing rivers and harbours immature worms, or microfilaria. In the human body, adult worms grow over a period of years, generating in turn more microfilaria which travel throughout the bloodstream. Infestation generally causes skin lesions and weight loss, but when the microfilaria travel to the eye, the resulting tissue damage causes blindness. About 340,000 people suffer from this extreme form of onchocerciasis, and in some Ghanaian villages 15 per cent of the population is permanently blind.

Two drugs, diethylcarbamazine and suramin, have been available for some time to kill microfilaria in the body, but both have severe side-effects and must be given often, making widespread use impossible in large and poor areas.

Control of onchocerciasis has thus centred on elimination of the blackfly, and a large-scale pesticide spraying programme, overseen by WHO, has operated since 1974 with some success. But without a treatment to kill the worms in the body, eradication seemed a forlorn hope.

Mectizan was developed by Merck in 1975 as a variant of ivermectin, a drug widely used against animal parasites. It kills microfilaria, and damages the repro- ductive system of the adult worms, putting a stop to infestation. Its great advantage is that it is effective in small doses given only once or twice a year, and has few sideeffects. There may be adverse effects on pregnant and lactating women and small children, but Dr Mohamed Aziz, who supervised the clinical development of Mectizan, says the doses necessary to control onchocerciasis are about one hundred times lower than the level at which reproductive problems are seen in mice.

The idea of developing ivermectin for human use originated with Dr Bill Campbell, a veterinary doctor at Merck, Sharpe and Dohme who had lived in Ethiopia. After some 'arm-twisting' by Campbell and others, Merck agreed to begin work on clinical development. Aziz, who comes from Bangladesh, described the onchocerciasis programme as the "highlight of my life".

Eric Ottesen of the National Institutes of Health says that Merck deserves "an enormous amount of credit" for pushing the idea past some initial scepticism at WHO. The company will benefit by good public relations from the drug giveaway, but the project will certainly cost it money. Dr Roy Vagelos, Merck's chairman, said the cost would not amount to a significant fraction of Merck's running costs. Although Mectizan, because of its potency and safety, could in principle command a high price, Merck could not neccessarily make much profit from it because the only countries that need it have no money.

Until recently, US law would not have countenanced even the giveaway of Mectizan, as it has not been approved for human use by the Food and Drug Administration (FDA). But US law now permits export of unapproved drugs provided the recipient allows it, and in this case the countries concerned are unlikely to say no, especially as the French Directorate of Pharmacy and Drugs has approved Mectizan for human use

Dr Halfdan Mahler, director-general of

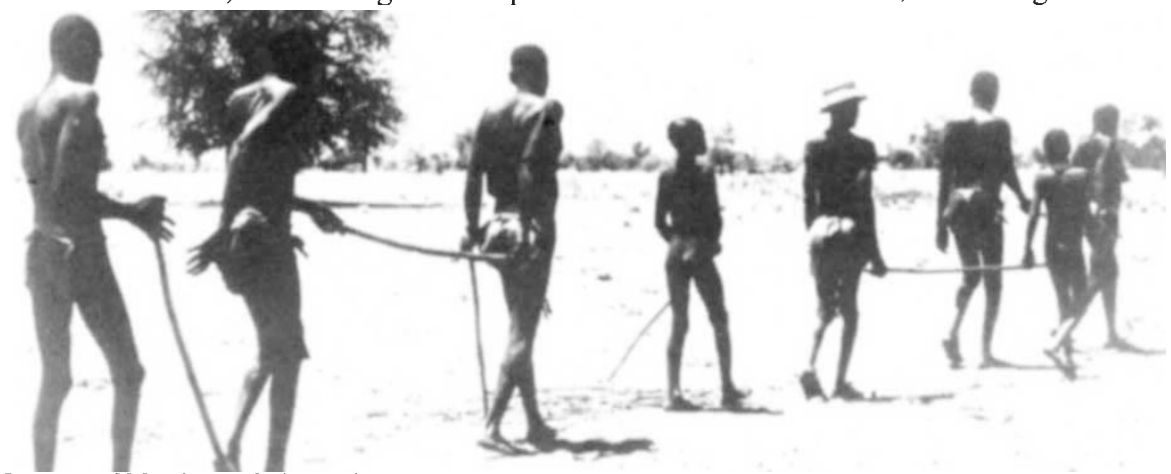

Groups of blind people being led by children. As many as 15 per cent of a village can be affected by river blindness.
Bhopal aftermath re-assessed

\section{New Delhi}

A REPORT just released by the Indian Council of Medical Research (ICMR) shows that fears of extensive damage to the health of survivors of the December 1984 tragedy may be misplaced.

Except for lung damage and associated disabilities, the methyl isocyanate (MIC) gas released from the Union Carbide pesticide factory had no major effect on survivors of the worst-ever industrial accident in India, which killed 2,000 people.

The studies were completed two years ago, but publication was withheld for fear of influencing the case now before the Bhopal court. The report is the first batch of ten research papers (out of the hundred or so received by ICMR) to be cleared by the government's legal experts. The remaining papers will be released later.

The ICMR launched some 30 investigations immediately after the accident. A major finding of these studies is lung damage and pronounced muscle weakness in many of the 10,700 people who needed hospital treatment. But lung function was severely impaired in only a few cases. One study noted that the number of "victims who had recognizable neurological syndromes was surprisingly low considering the magnitude of exposure to the toxic gas". Another study said that "any chromosomal aberrations found were minor". No mutagens were detected in urine, and no significant changes were found in human placentas. Suggestions that MIC could induce sterility in males were discounted by studies that showed no alterations in sperm count, mobility and morphology.

The ICMR studies did conclude, however, that long-term treatment might be necessary for people with lung damage who can no longer work. Prolonged psychotherapy is also proving necesssary for nearly a quarter of the survivors who are showing signs of anxiety and neurotic depression.

K.S.Jayaraman

WHO, joined in the chorus of praise for Merck's "generous gesture", and hoped that it would help break down the "paranoia" that exists between WHO and the pharmaceutical industry. WHO has $\$ 4$ million available to begin to set up the infrastructure for delivery, but will need more. Mahler hopes that the promise of a manifestly effective and relatively cheap programme such as this will persuade member countries that WHO is not the "bloated bureaucracy" that many think it. Mahler foresees visible reduction in the incidence of river blindness in a few years, and maintains that eradication of the disease by the end of the century is a reasonable goal.

David Lindley 